核融合研究第 7 巻第 6 号 1961 年 12 月

\title{
プラズマの境界層 $\mathbb{I}^{*}$ \\ 一 Maxwe11 分布の場合 -
}

伏。見康治 ブラスマ研)

矢島，信 男 (プラズマ研)

都 築 俊: 夫( 阪大理)

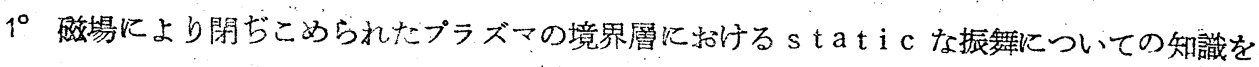
与えるマックスウェル方程式は、前の報告 ${ }^{(1)}$ で与えた。空間座蹯 $x$ 点に拈ける、ベクトル及びス タチックポテンシャルを夫々 $\mathrm{A}(x), \Phi(x)$ とし、この点に批る粒子圧を $\mathrm{P}(\mathrm{A}, \Phi)$ と すると

$$
\left\{\begin{array}{l}
\frac{\mathrm{d}^{2} \Phi}{\mathrm{d} x^{2}}=\frac{1}{\varepsilon} \frac{\partial \mathrm{P}}{\partial \Phi} \\
\frac{\mathrm{d}^{2} \mathrm{~A}}{\mathrm{~d} x^{2}}=-\mu \frac{\partial \mathrm{P}}{\partial \mathrm{A}}
\end{array}\right.
$$

である。ここで

$$
\begin{aligned}
& \mathrm{P}(\mathrm{A}, \bar{\Phi})=\int 2 \mathrm{mU}(x) \mathrm{n}(\overrightarrow{\mathrm{u}}) \mathrm{ud}^{3} \mathrm{u} \\
& \mathrm{U}^{2}(x)=\mathrm{u}^{2}-\frac{2 \mathrm{e}}{\mathrm{m}} \Phi(x)+\frac{2 \mathrm{e}}{\mathrm{m}} \mathrm{vA}(x)-\frac{\mathrm{e}^{2}}{\mathrm{~m}^{2}} \mathrm{~A}^{2}(x)
\end{aligned}
$$

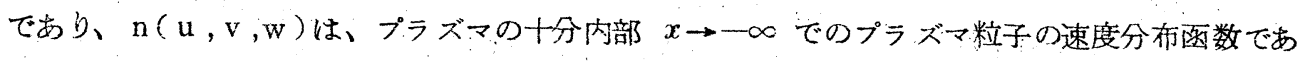
る。又積分領域は $x \geq x^{\prime}>-\infty$ のすべての $x^{\prime}$ に対してU( $\left.x^{\prime}\right)>0$ である。

(1)の矛一積分は

$$
\frac{1}{2 \mu}\left(\frac{\mathrm{dA}}{\mathrm{d} x}\right)^{2}-\frac{\varepsilon}{2}\left(\frac{\mathrm{d} \Phi}{\mathrm{d} x}\right)^{2}+\mathrm{P}(\mathrm{A}, \Phi)=\mathrm{cons} \mathrm{t} \equiv \mathrm{c}
$$

* The boundary layer of plasma II

- Maxwell volocity distribution -

※ K. Hus imi, N. Yajima

(Institute of Plasme Physics, Nagoya University)

T. Tsuzukj (Department of Physics, Osaka University) 
伏見. 矢島 - 都築

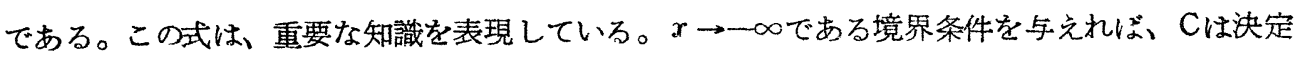

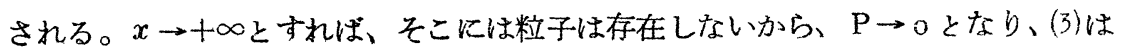

$$
\frac{1}{2 \mu}\left(\frac{\mathrm{dA}}{\mathrm{d} x}\right)_{\infty}^{2}-\frac{\varepsilon}{2}\left(\frac{\mathrm{d} \Phi}{\mathrm{d} x}\right)_{\infty}^{2}=\mathrm{C}
$$

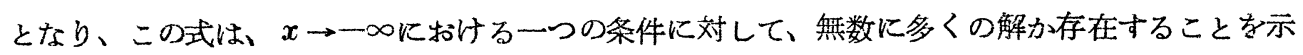
している。neutral confinement と云5ことは、それらの5ちの特别な一つの場合に すぎない。しかもそれらの解林、ブラズマの内部に行くに従って、ある一つの形に近ずいてゆく。 このことは、Thomas-Fermi の原子模型の場合と全く同じ事情になっている。原子の内部 のポテンシャル函数は、その原子が中性であるか、イオンであるかには関係しない。このよ5に、

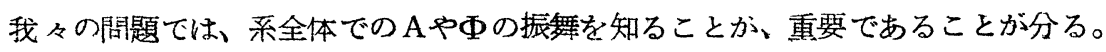

以上のことは、この間題に対して Sommerfeld の、Thomas -Ferm 1 原子模型に" $z^{2}$ する優れた論交 ${ }^{(2)}$ の手法を応用出来るのではないかと云らことを示している。我々は彼の方法を 用いる。

最後に、最近の論文にふれておこち。Grad ${ }^{(3)}$ はややは同時問題を論じている。しかし、彼 は問題をあまりに簡単化したために( 1 の と e lectron の mass defference を 無視した)、charge separationを無視してしまっている。従って、彼に対しても Harris や Tonks に刘すると同じ批判が成立する。又理想化された場合として、 mas defference を無視することを認めるとしてひ、彼の preprint のなかの定理は誤 つ ている。彼は、般に速度分布函数か、粒子のエネルギ一たけに依存して、速度の方向に依存せ ボ、かつ trapped particle がないときには、プラズマの十分内部での“境界面”に 平行な電流 Jyは、vector potentral Aに比例すると主張する。しかし、例えば三次 元マ,クスウェル分布 $\exp \left[-\mathrm{m}\left(\mathrm{u}^{2}+\mathrm{v}^{2}+\mathrm{w}^{2}\right) / 2 \mathrm{RT}\right]$ を持つ場合について計算してみれ ば分るよ5に Jyは $\mathrm{A}^{1 / 2}$ に比例する。

$2^{\circ}$ 我々の方法は、一般的な速度分布の場合に搪張することは簡単なので、プラズマか十分内 部 $(x \rightarrow-\infty)$ でマノクスウェル速度分布 (一次元)

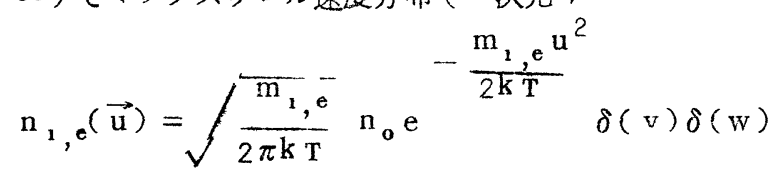

を持つ場合について論じる。無次元の量 


$$
\begin{aligned}
& \text { プラズマの境界層II-Maxwe } 11 \text { 分布の場合一 } \\
& \varphi \equiv \frac{\mathrm{e}}{\mathrm{kT}} \Phi, \quad \mathrm{a}=\frac{\mathrm{ec}}{\mathrm{kT}} \mathrm{A}
\end{aligned}
$$

を導入する。積分領域に trapped region が現われない、即ち $\varphi-\frac{1}{2} \lambda a^{2}$ 及び $\varphi+\frac{1}{2} \lambda k \mathrm{a}^{2}$ が正の増大函数とすると、粒子王は

$$
\mathrm{P}(\mathrm{a}, \varphi) / \mathrm{kT}=\mathrm{e}^{\varphi-\frac{1}{2} \lambda \mathrm{a}^{2}}+\mathrm{e}^{-\varphi-\frac{1}{2} \lambda_{\kappa} \mathrm{a}^{2}}
$$

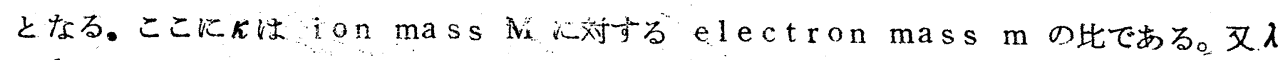
$={ }^{\mathrm{kT}} / \mathrm{mc}^{2}$ 。長さの単位としてDebye長を導入する。

$$
x=\lambda_{D}, \quad \lambda_{\mathrm{D}}^{-]}=\sqrt{\frac{\mathrm{n}_{\mathrm{o}} \mathrm{e}^{2}}{\varepsilon \mathrm{kT}}}
$$

マックスウェルカ程式は、 $\xi$ の微分をdotで示して

$$
\left\{\begin{array}{l}
\| \prime=\mathrm{e}^{\varphi-\frac{1}{2} \lambda \mathrm{a}^{2}}-\mathrm{e}^{-\varphi-\frac{1}{2} \lambda \kappa \mathrm{a}^{2}} \\
\mathrm{a}=\lambda \mathrm{a}\left(\mathrm{e}^{\varphi-\frac{1}{2} \lambda \mathrm{a}^{2}}+\kappa \mathrm{e}^{-\varphi-\frac{1}{2} \lambda \kappa \mathrm{a}^{2}}\right)
\end{array}\right.
$$

となる。境界条件は

$$
\varphi=\dot{\varphi}=\mathrm{a}=\dot{\mathrm{a}}=0 \quad \text { for } x \rightarrow-\infty
$$

及び

$$
\begin{aligned}
& \varphi \rightarrow \infty, \dot{\varphi}=\text { finite }=\varepsilon \\
& \mathrm{a} \rightarrow+\infty, \dot{\mathrm{a}}=\mathrm{f} \text { inite }=\mathrm{b}
\end{aligned}
$$

である。(10)を用いると为一積分(3)は

$$
\frac{1}{2} \dot{\mathrm{a}}^{2}-\frac{1}{2} \dot{\varphi}^{2}+\mathrm{e}^{\varphi-\frac{1}{2} \lambda \mathrm{a}^{2}}+\mathrm{e}^{-\varphi-\frac{1}{2} \lambda \kappa \mathrm{a}^{2}}=2
$$

となり、これに(11)を入れると、 $\varepsilon$ bの関係

$$
b^{2}-\varepsilon^{3}=4
$$

を5る。 
伏見・矢皂・都築

ところで、(12)より系の Lagrangian Lは

$$
\mathrm{L}=\frac{1}{2} \dot{\mathrm{a}}^{2}-\frac{1}{2} \dot{\varphi}^{2}-\mathrm{e}^{\varphi \frac{1}{2} \lambda \mathrm{a}^{2}}-\mathrm{e}^{-\varphi \frac{1}{2} \lambda \kappa \mathrm{a}^{2}}
$$

だから、 a， $\varphi$ に共役な運野量は

$$
\begin{aligned}
& \mathrm{pa}=\frac{\partial \mathrm{L}}{\partial \dot{\mathrm{a}}}=\dot{\mathrm{a}}, \\
& \mathrm{p} \varphi=\frac{\partial \mathrm{L}}{\partial \phi}=-\dot{\varphi}
\end{aligned}
$$

だから Hamiltonian H は

$$
\mathrm{H}=\frac{1}{2} \mathrm{pa}{ }^{2}-\frac{1}{2} \mathrm{p} \varphi^{2}+\mathrm{e}^{\varphi-\frac{1}{2} \lambda \mathrm{a}^{2}}+\mathrm{e}^{-\varphi-\frac{1}{2} \lambda \kappa \mathrm{a}^{2}} .
$$

である。従って、(9) と等価な方程式は

$$
\left\{\begin{array}{l}
\dot{\mathrm{a}}=\frac{\partial \mathrm{H}}{\partial \mathrm{p}}=\mathrm{p}_{\mathrm{a}} \\
\dot{\varphi}=\frac{\partial \mathrm{H}}{\partial \mathrm{p}}=-\mathrm{p}_{\varphi} \\
\dot{\mathrm{p}}_{\mathrm{a}}=-\frac{\partial \mathrm{H}}{\partial \mathrm{a}}=\lambda \mathrm{a}\left(\mathrm{e}^{\varphi-\frac{1}{2} \lambda \mathrm{a}^{2}}+\kappa \mathrm{e}^{-\varphi-\frac{1}{2} \lambda \kappa \mathrm{a}^{2}}\right) \\
\dot{\mathrm{p}}_{\varphi}=-\frac{\partial \mathrm{H}}{\partial \varphi}=-\mathrm{e}^{\varphi-\frac{1}{2} \lambda \mathrm{a}^{2}}+\mathrm{e}^{-\varphi-\frac{1}{2} \lambda \kappa \mathrm{a}^{2}}
\end{array}\right.
$$

となる。我々は、この方程式基礎にして、 $\mathrm{a} \ll 1$ 及び $\mathrm{a} \gg 1$ にいて解き、内接法により、 全系の解を近似的に求める。

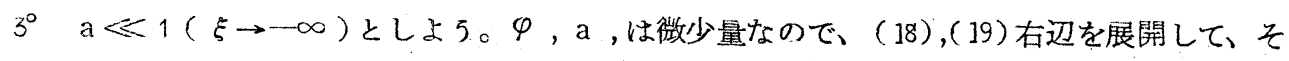
の初項のみを取る。(15) 〜 (19) から

$$
\left\{\begin{array}{l}
\frac{\mathrm{d} \varphi}{\mathrm{da}}=-\frac{\mathrm{p}_{\varphi}}{\mathrm{p}_{\mathrm{a}}} \\
\frac{\mathrm{dp} \varphi}{\mathrm{dp} a}=\frac{\frac{1}{2} \lambda(1-\kappa) \mathrm{a}^{2}-2 \varphi}{\lambda(1+\kappa) \mathrm{a}}
\end{array}\right.
$$

を5る。 $\mathrm{p}_{\varphi}$ も $\mathrm{p}_{a}$ も敩少量なので、 $\beta, \gamma$ を定数として 
プラズマの境界層 II-Maxwe 11 分布の場合一

$$
\begin{aligned}
& \frac{\mathrm{d} \varphi}{\mathrm{da}} \approx \beta \frac{\varphi}{\mathrm{a}} \\
& \frac{\mathrm{dp} \varphi}{\mathrm{d} \mathrm{p}_{\mathrm{a}}} \approx \gamma \frac{\mathrm{p}_{\varphi}}{\mathrm{p}_{\mathrm{a}}}
\end{aligned}
$$

とおくと、(20)より

$$
\varphi=\frac{\lambda(1-\kappa) a^{2}}{2\{2-\beta \gamma(1+\kappa)\}}
$$

を5る。(21)より、 $\beta=\gamma=2$ なることは明らかだから

$$
\varphi=\frac{\lambda(1-\kappa)}{4\{1-2 \lambda(1+\kappa)\}} \mathrm{a}^{2}
$$

を5る。同様にすれば、 $\mathrm{p}_{\mathrm{a}}, \mathrm{p} \varphi$ をa の函数として表わせて

$$
\begin{aligned}
& \mathrm{p}_{\mathrm{a}}=\sqrt{\lambda(1+\kappa)} \mathrm{a} \\
& \mathrm{p} \varphi=-\frac{\dot{\lambda}^{3 / 2}(1-\kappa) \sqrt{1+\kappa}}{2\{1-2 \lambda(1+\kappa)\}} \mathrm{a}^{2}
\end{aligned}
$$

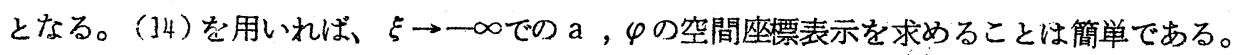

$4^{\circ}$ 次に $\mathrm{a} \gg 1(\xi \rightarrow+\infty)$ での漸近解を求めよ5。(11)より、 $\mathrm{p}_{\mathrm{a}}, \mathrm{p}_{\varphi}$ は $\xi \rightarrow+\infty て ゙$ $\mathrm{p}_{\mathrm{a} \infty}=\mathrm{b}, \mathrm{p}_{\varphi_{\infty}}=-\varepsilon$ K近ずくのでこの值からのずれを $\mathrm{X}, \mathrm{Y}$ とする。 $\mathrm{X}, \mathrm{Y}$ は $\mathrm{a} \rightarrow \infty て ゙$ 零 となる。

$$
\left\{\begin{array}{l}
\mathrm{p}_{\mathrm{a}}=\mathrm{p}_{\mathrm{a} \infty}+\mathrm{X} \\
\mathrm{p}_{\varphi}=\mathrm{p}_{\varphi \infty}+\mathrm{Y}
\end{array}\right.
$$

(16),(17)より、 $\varphi$ をaで表わしたときの、初めの項は

$$
\varphi=\alpha \mathrm{a}+\mathrm{F}
$$

となる。ここに $\propto \equiv-\mathrm{P}_{\varphi_{\infty}} / \mathrm{P}_{\mathrm{a} \infty} ， \mathrm{~F}$ は定数である。そこで $\varphi$ の上の值よりのずれを一 Zと 报くと 


$$
\varphi=\alpha \mathrm{a}+\mathrm{F}-\mathrm{Z}
$$

$\mathrm{X}, \mathrm{Y}, \mathrm{Z}$ は微少量である。

ここで次の近似をする。即ち、境界層の外の方では、プラズマは、卧とんどイオンばかりであ り、電子の影響は無視出来る。このよ5にすれば、(16)〜 (19) 出、

$$
\left\{\begin{array}{l}
\dot{\mathrm{a}}=\mathrm{P}_{\mathrm{a} \infty}+\mathrm{X} \\
\dot{\mathrm{Z}}=\alpha \mathrm{X}+\mathrm{Y} \\
\dot{\mathrm{X}}=\lambda \kappa \mathrm{a} \mathrm{e}^{-\alpha \mathrm{a} \frac{1}{2} \lambda \kappa \mathrm{a}^{2}-\mathrm{F}} \\
\dot{\mathrm{Y}}=\mathrm{e}^{-\alpha \mathrm{a}-\frac{1}{2} \lambda \kappa \mathrm{a}^{2}-\mathrm{F}}
\end{array}\right.
$$

となる。(26),(28)より直ちに

$$
\mathrm{x}=\frac{\lambda \kappa}{\mathrm{p}_{\mathrm{a} \infty}} \int_{\infty}^{\mathrm{a}} \mathrm{a} \mathrm{e}^{-\alpha 2-\frac{1}{2} \lambda \kappa \mathrm{a}^{2}-\mathrm{F}} \mathrm{da}
$$

を5る。部分積分をして、その初項を取ると

$$
\mathrm{X} \simeq-\frac{1}{\mathrm{p}_{\mathrm{a} \infty}} \mathrm{e}^{-\alpha \mathrm{a}-\frac{1}{2} \lambda \kappa \mathrm{a}^{2}-\mathrm{F}}
$$

となる。 $(28)(29)(30) よ り$

$$
\mathrm{Y}=\frac{1}{\lambda \kappa \mathrm{a}} \mathrm{X} \simeq-\frac{1}{\lambda_{\kappa} \mathrm{p}_{\mathrm{a} \infty \mathrm{a}}} \mathrm{e}^{-\alpha \mathrm{a}-\frac{1}{2} \lambda \kappa \mathrm{a}^{2}-\mathrm{F}}
$$

$(27)(28)(30)$ よb

$$
\mathrm{z} \simeq \frac{\lambda \kappa \alpha \mathrm{a}+1}{\lambda^{2} \kappa^{2} \mathrm{p}_{\mathrm{a} \infty \mathrm{a}^{2}}^{\mathrm{a}^{2}}} \mathrm{e}^{-\alpha \mathrm{a}-\frac{1}{2} \lambda \kappa \mathrm{a}^{2}-\mathrm{F}}
$$

を5る。(32)を(25)に代入すれば

$$
\varphi=\alpha \mathrm{a}+\mathrm{F}-\frac{\lambda \kappa \alpha \mathrm{a}+1}{\lambda^{2} \kappa^{2} \mathrm{p}_{\mathrm{a} \infty \mathrm{a}^{2}}^{\mathrm{a}^{2}}} \mathrm{e}^{-\alpha \mathrm{a}-\frac{1}{2} \lambda \kappa \mathrm{a}^{2}-\mathrm{F}}
$$

となる。(32)(33)の分数部分の 1 の項は むし neutral confinement $\alpha=0$ を考 㝋るときには、初項となる。 
$5^{\circ}$ 内接法により両極端での)解 (22),(33)をつなご5。簡単な函数として

$$
\varphi=\frac{\beta \mathrm{a}^{2}(\alpha \mathrm{a}+\mathrm{F})^{2}}{\gamma \mathrm{a}^{2}(\alpha \mathrm{a}+\mathrm{F})+\delta(\lambda \kappa \alpha \mathrm{a}+1) \mathrm{e}^{-\alpha \mathrm{a}-\frac{1}{2} \lambda \kappa \mathrm{a}^{2}}}
$$

ととる。 $\beta, \gamma, \delta$ は $\rightarrow+\infty$ で(33)に $\mathrm{a} \rightarrow 0$ で(22)につながるよ5に決める。その結果 は

$$
\varphi=\frac{\lambda(1-\kappa) \mathrm{a}^{2}(2 \alpha+\mathrm{F})^{2}}{\lambda(1-\kappa) \mathrm{a}^{2}(\alpha \mathrm{a}+\mathrm{F})+4 \mathrm{~F}^{2}\{1-2 \lambda(1+\kappa)\}(\lambda \kappa \alpha \mathrm{a}+1) \mathrm{e}^{-\alpha \mathrm{a}-\frac{1}{2} \lambda \kappa \mathrm{a}^{2}}}
$$

と、条件式

$$
F^{2} e^{F}=\frac{1-\kappa}{4 \lambda \kappa^{2} P_{a \infty}^{2}\{1-2 \lambda(1+\kappa)\}}
$$

である。

ここでFの意味を調べよ5。（26）と（30）から、を方で表わすと

$$
\mathrm{a}=\mathrm{p}_{\mathrm{a} \infty}\left(\xi-\xi^{*}\right)+\frac{1}{\lambda \kappa \mathrm{p}_{\mathrm{a} \infty}^{3}\left(\xi-\xi^{*}\right)} \mathrm{e}^{-\alpha \mathrm{p}_{\mathrm{a} \infty}\left(\xi-\xi^{*}\right)-\frac{1}{2} \lambda \kappa \mathrm{p}_{\mathrm{a} \infty}^{2}\left(\xi-\xi^{*}\right)^{2}-\mathrm{F}}
$$

となり、これは、解が translational invariant であることを示している。小さ い補正項を無視すれば

$$
\varphi=-\mathrm{p} \varphi_{\infty}^{\xi}+\varphi_{\infty}
$$

だから

$$
\mathrm{F}=\varphi_{\infty}-\mathrm{p} \varphi_{\infty} \xi^{*}
$$

となり、結局Fは translation に対する不変量と云5ことになる。特に neutral confinement を考えるとされは $P_{\varphi \infty}=0$ だから、原点のえらび方に無関係に stat ic potential は一定值

$$
\varphi_{\infty}^{2} e^{\varphi_{\infty}}=\frac{1-\kappa}{16 \lambda \kappa^{2}\{1-2 \lambda(1+\kappa)\}}
$$

に飽和する。 


$$
\text { 伏見 - 矢島 - 都築 }
$$

(34)と(16) 〜 (19)を用いれば、vector 及び static potential 及び電場・磁 場の $\xi$ 表示がえられる。我々に特に興味ある場合、即ち neutral confinement $\left(\mathrm{p}_{\varphi_{\infty}}\right.$ $\left.=0, \mathrm{p}_{\mathrm{a} \infty}=2\right)$ の場合について、数值的に積分した結果はグラフに示す通りである。 $\varphi_{\infty}$ の值 はtypical な温度に対して(37)より求めた。

$\mathrm{T}=10 \mathrm{KeV}$ に対して

$$
\begin{aligned}
& \varphi_{\infty}=11 \\
& \mathrm{e} \Phi_{\infty}=110 \mathrm{KeV}
\end{aligned}
$$

$\mathrm{T}=1 \mathrm{KeV}$ に対して

$$
\begin{aligned}
& \varphi_{\infty}=13 \\
& \mathrm{e} \Phi_{\infty}=13 \mathrm{KeV}
\end{aligned}
$$

このよ5に、static potential の飽和值は、プラズマの温度の約10倍である。これら のグラフから明らかなことは

（1）諸量の変化の椂子は温度によってほとんど変らない。

（2）電場が最小值をとる点は、 electron rich region から ion rich region に5つる local neutral な点である。この点より外側では磁場は外部磁場の大ささ にほとんど等しい。このことは i on の運勤は電流にはほとんど奇与しないことを示してい る。

（3）電場のグラフと合せ考光れば、 e l e c t r on は主に磁場により、i on は主に電場によ り反撥される。

（4）磁場の值の $1 / \mathrm{e}$ 巾で境界缯の厚みを定義すると、約 8 electron Larmor 半径で ある。この值は shock の方で云われている、1 on と e lectron の Larmor 半 径の幾何平均

$$
\sqrt[4]{\frac{M}{m}} \sqrt{\frac{m}{2 n_{o} e^{2} \mu}}=\frac{1}{\sqrt{\pi}} \quad \lambda_{p}
$$

と数値的には等しい。

（5）電気的二重層の厚さも電場の $1 / \mathrm{e}$ 巾で定義すれば約 7 e lectron Larmor 羘径であ る。 


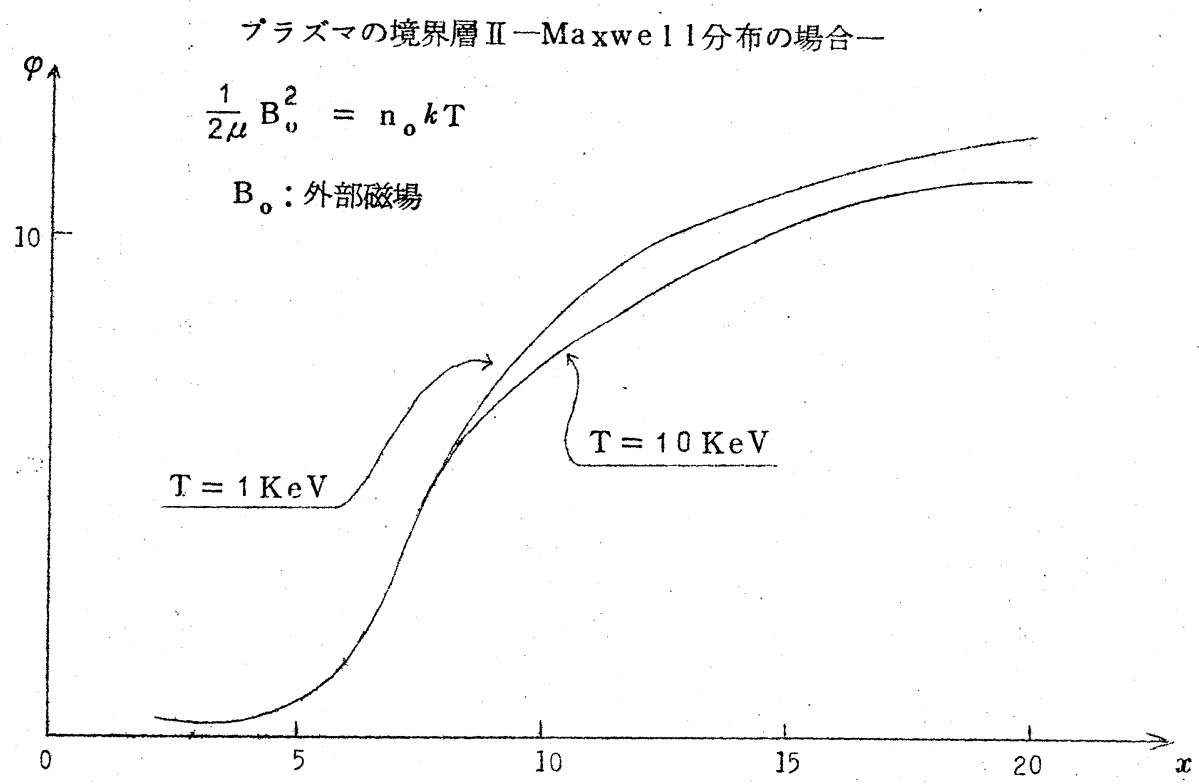

長さの単位は電子ラーマー半径 $\sqrt{\frac{m}{2 n_{0} e^{2} \mu}}$

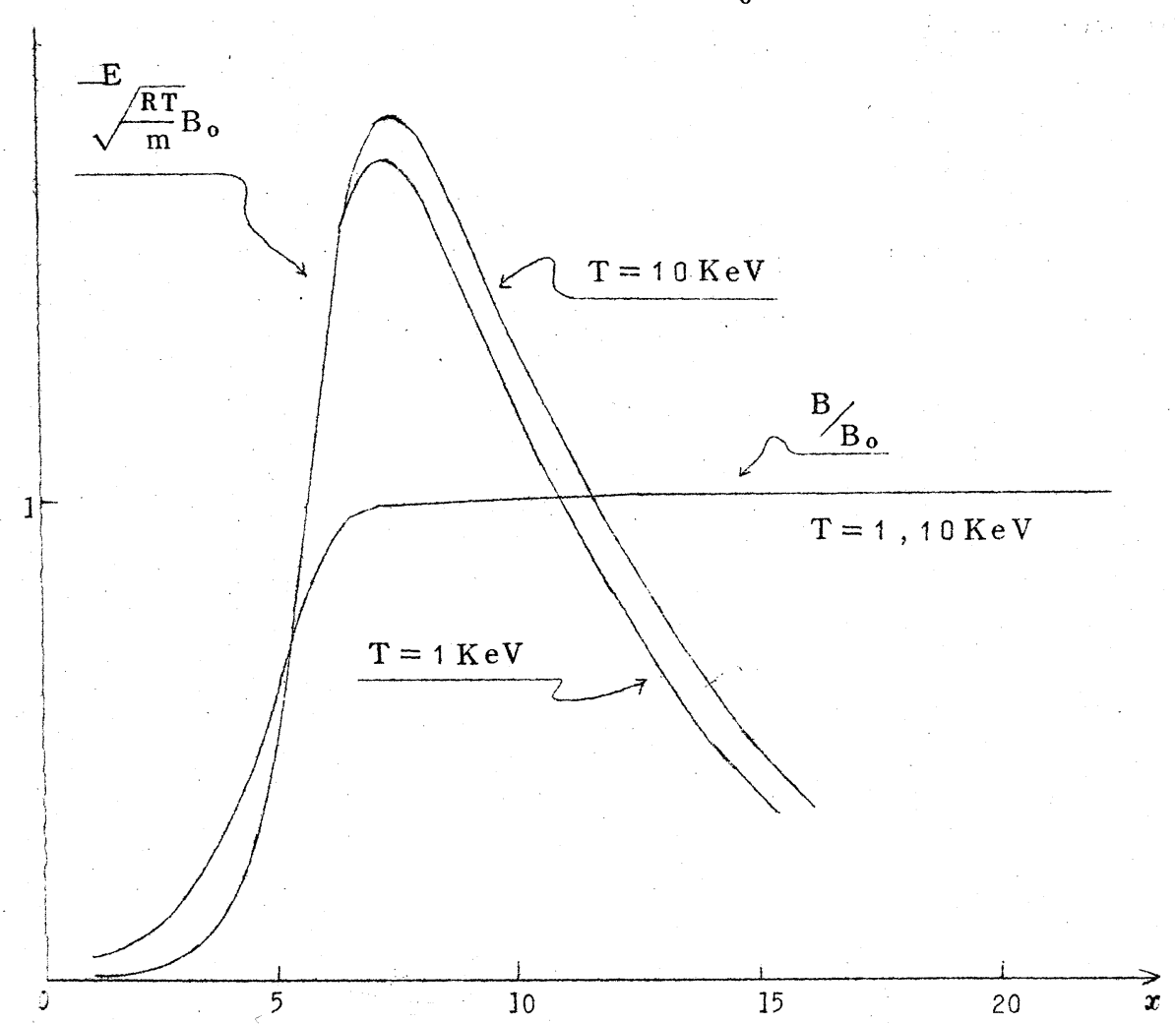




$$
\begin{gathered}
\text { 伏見 矢島 自都築 } \\
\text { 参 考 文 献 }
\end{gathered}
$$

（1）伏見·矢島·都築 核融合研究 白 $137(1961)$

K. Husimi, N. Yajima and T. Tsuzuki

"On the Structure of a Fresh Plasma Surface"

(2) A. Sommerfeld ZS. f. Phys. $78 \quad 283$ (1932)

(3) H. Grad NYO - 9491

"The Boundary Layer Between a Plasma and a Magnetic Fi e ld - I" 